\title{
Der phänomenographische Ansatz in der glottodidaktischen Forschung
}

\author{
The phenomenographic approach \\ in research on second language learning
}

\begin{abstract}
The phenomenographic approach in educational research dates back to the early1980s. Marton and his co-workers focused on the significant role which is played by the individual beliefs of learners in the processes of teaching and learning. The study of learning experiences, reflections on these experiences and verbalization of these reflections have defined a new perspective in the perception of educational reality. The paper presents the basic assumptions of the phenomenographic approach and its application in the study of foreign language learners' beliefs. Since beliefs result from many factors, among others of a socio-cultural nature, particular attention is paid to the dynamics of how beliefs are shaped in the light of the Activity Theory proposed by Vygotsky and his successors Leontiev and Engerström. The paper finishes with a discussion on the prospects of the study of beliefs in the Polish educational context, with particular consideration of foreign language philology students.
\end{abstract}

KEYWORDS: second language research, learner beliefs, phenomenography, sociocultural theory. SCHLÜSSELWÖRTER: Fremdsprachenforschung, Lernermeinungen, Phänomenographie, soziokultureller Ansatz.

\section{EINFÜHRENDE BEMERKUNGEN}

In der qualitativen Sozialforschung wird der Standpunkt vertreten, dass die etische (äußere) Perspektive des Zugangs an das Erkenntnisobjekt (von der Seite der Forschenden aus) durch die emische (innere) Sichtweise der Erkenntnissubjekte (der Erforschten) ergänzt werden soll. Rein etische oder emische Forschungsansätze bergen nämlich, gerade durch die Art ihrer Herangehensweise, die Gefahr in sich, relevante Erkenntnisse außer Acht zu lassen: aus der rein etischen Perspektive können die individuellen Positionen der Erforschten von der forschenden Person nicht unbedingt theoretisch vorweggenommen und in Folge ausgeblendet werden; die eingenommene rein emische Perspektive kann dagegen durch mangelnde Distanz der Erforschten zum Untersuchungsgegen- 
stand zu wenig generalisierbaren Erkenntnissen führen und diese stark „ethnozentrisch" erklären (vgl. Schramm \& Schwab 2016: 142; Deters-Philipp 2018: 173).

In der Fremdsprachenforschung ist man sich seit Langem im Klaren, dass die Einbeziehung der Lernerperspektive ins Untersuchungsdesign eine wichtige, in manchen Fällen unabdingbare Voraussetzung für die Erklärung der Lernprozesse bildet. Diese Forderung leitet sich vor allem von der Spezifik der Lernprozesse, hier der Fremdsprachenlernprozesse, ab: Daten, wie Lernende beispielsweise eine neue Sprache wahrnehmen, verarbeiten, abspeichern, abrufen und gebrauchen, welche Entscheidungen (etwa grammatisch-lexikalischer Art) sie treffen, lassen sich aus der Außenperspektive nur partiell erheben und auswerten. Die Nachvollziehung der Lernersprache kann dafür ein Beispiel sein, wenn man Lernende bei der Produktion der Sprachäußerungen beobachtet und dabei bestimmte Probleme bemerkt, aber manchmal schwer einzuschätzen vermag, worauf diese zurückzuführen sind. Die innere Sichtweise ermöglicht daher, etwa durch intro- oder retrospektive Verfahren (auch als Lautes Denken bezeichnet), neue Perspektiven aufzudecken und subjektive Erklärungen der Erforschten kennen zu lernen. Natürlich muss an dieser Stelle nachdrücklich hervorgehoben werden, dass die Lernerperspektive in Anbetracht der Komplexität der Sprachlernprozesse und der ganzen menschlichen Kognition auch als eine Außensicht zu betrachten ist, die - wie gerade oben festgestellt - mehr oder weniger subjektiv die erforschte Wirklichkeit erkennt und deutet.

Die Subjektivität ist ein kennzeichnendes Merkmal der Meinungen, Überzeugungen, Erklärungsweisen konkreter Lernender mit ihren individuellen Sprachlernerfahrungen und sozialem Hintergrund. Um tiefgreifender die Sprachlernprozesse erläutern zu können, ist von Relevanz zu erfahren, wie Lernende selbst diese Prozesse wahrnehmen, wie sie sich diese erklären, wie sie ihre Sprachlerngeschichten nachvollziehen und welchen Wert unterschiedlichen Faktoren beimessen, schließlich, oder in erster Linie - weil dies als einer der Entscheidungspunkte in vielerlei Hinsicht (z. B. bei der Anwendung von Lern-, Kommunikationsstrategien) gelten kann - wie Lernende das Phänomen Sprache verstehen und welche Rolle(n) ihr (etwa beim Lernen, im Gebrauch, im zwischenmenschlichen Mit- und Nebeneinander) zuschreiben. Dies sind nur einige ausgewählte Forschungsprobleme, die die Sprachlehr- und -lernforschung seit den 1990er-Jahren aufgenommen hat. Exemplarisch können an dieser Stelle das Forschungsprogramm Subjektive Lernertheorien, das vor allem in Deutschland fokussiert wurde (z. B. Kallenbach 1996; Grotjahn 1998; Klippel 2013; Vidgren 2018, vgl. auch Rück 2009), oder aber unterschiedliche internationale Studien zum Problem von Learner Beliefs genannt werden, die besonders in qualitativer Hinsicht gewichtige Erkenntnisse geliefert und neue Fragestellungen (auch forschungsmethodischer Natur) aufgeworfen haben (zur Übersicht vgl. die 
Sammelbände von Kalaja \& Barcelos 2003; Kalaja, Barcelos, Aro \& Ruohotie-Lyhty 2016). Auch in der polnischen glottodidaktischen Forschungsarbeit wird die Lernerperspektive in mehreren quantitativ und / oder qualitativ ausgerichteten Untersuchungen herangezogen, um die Sichtweisen von polnischen Lernenden in unterschiedlichen Lernkontexten (etwa Schüler, Studenten, Studenten der Neuphilologien [auch als künftige Sprachlehrer], Senioren) zu verschiedenen Problemen des Sprachenlernens aufzugreifen (vgl. z. B. Pawlak 2008; Adamczak-Krysztofowicz 2009; Nerlicki 2011; Michońska-Stadnik 2013; Ellis 2014; Jaroszewska 2014; Piechurska-Kuciel 2014; Werbińska 2010, 2015; Aleksandrzak 2018). Den geäußerten Sichtweisen liegt reflexives Handeln der Lernenden zu Grunde, das sich durch die externe (etwa durch Fragestellungen in semistrukturierten Interviews) und / oder interne Evozierung der Bewusstheit in dem Was (was unterliegt der Reflexion?) und dem Wie (wie wird das Reflektierte versprachlicht, aber auch nonverbal geäußert?) der individuellen Meinungen manifesiert.

Die Berücksichtigung der Lernerperspektive in der Erforschung der Bildungsprozesse hat in der pädagogischen, psychologischen und soziologischen Forschungspraxis eine lange Tradition. Einer der Ansätze, der viele Untersuchungen theoretisch und methodisch untermauert, ist der phänomenographische Ansatz. Vor allem in der Lernpsychologie stößt er bereits seit den 1970er-Jahren auf große Resonanz. In der Fremdsprachenforschung dagegen wurde er - mit einigen einzelnen theoretischen und empirischen Ausnahmen - bisher fast kaum rezipiert, obwohl er viele Paralellen zur Erforschung der Meinungen von Fremdsprachenlernenden aufweist und, was ohne Zweifel besonders herauszuheben ist, ein neues Licht auf die thematisierte Untersuchungswirklichkeit wirft.

Drei Ziele werden in diesem Aufsatz verfolgt: Zuerst werden die Grundannahmen des phänomenographischen Ansatzes, vor allem aus der Sicht der pädagogischen Forschung, dargestellt (Punkt 2). Danach wird auf ausgewählte Diskussionsbeiträge zugegriffen, die den phänomenographischen Ansatz bei der Erforschung fremdsprachlicher Lernprozesse thematisieren (Punkt 3). Schließlich wird überlegt, welche theoretischen und empirischen Perspektiven der phänomenographische Ansatz für weitere Untersuchungen, insbesondere im polnischen Bildungskontext eröffnet (Punkt 4).

\section{PHÄNOMENOGRAPHIE IN DER PÄDAGOGISCHEN FORSCHUNGSTRADITION}

Begründer des phänomenographischen Ansatzes ist der schwedische Lernpsychologe und Pädagoge Ference Marton, der mit seiner Forschergruppe von der Universität Göteborg bereits in den 1970er-Jahren die theoretischen Grund- 
sätze ausgearbeitet hat. In dem heute als richtungsweisend geltenden Aufsatz Phenomenography - decribing conceptions of the world about us (1981) überlegte er eine einfache Frage, warum einige Kinder besser und leichter als andere lernen. Dieser Frage stellte er gleich das von Säljö (1981) etwas anders formulierte Problem gegenüber: Was halten die Menschen davon, dass einige Kinder besser und leichter als andere lernen? (Marton 1981: 177f.). Diese zweite Frage liegt in ihren allgemeinen Konturen der ganzen phänomenographischen Auseinandersetzung mit der Wahrnehmung und Deutung der Wirklichkeit zu Grunde. Das primäre Ziel der Phänomenographie ist nämlich ", to find and systematize forms of thought in terms of which people interpret aspects of reality (Marton 1981: 180). Die Bezeichnung Phänomenographie geht auf griechische Wörter zurück: phainómenon (Erscheinung) und graphein (Beschreibung). Der Ansatz steht in enger Beziehung zur Husserlschen Phänomenologie. Während in der Phänomenologie die forschende Person das eigene Bewusstsein und ein bestimmtes Phänomen als etwas, was unabhängig von dem Bewusstsein existiert, einer Analyse unterzieht, zielt die Phänomenographie darauf ab, dieses Phänomen (als nur im Bewusstsein existierend) in unterschiedlich ausgeprägten Varianten (näher siehe unten) bei anderen Menschen zu erfassen (vgl. Kossobucka 2013: 409; Szostkiewicz 2018: 165).

Phänomenographie und Phänomenologie treffen sich also in ihrem gemeinsamen Forschungsgegenstand insofern, als beide danach streben, Eigenschaften menschlichen Erlebens und menschlichen Bewusstseins aufzudecken (Marton \& Booth 2014: 182).

Wenn die Forschersicht auf das zu untersuchende Erkenntnisobjekt als Perspektive des ersten Grades bezeichnet wird, kommt dem Lernenden in seiner Erfassung der Realität die Perspektive des zweiten Grades zu (vgl. auch Jurgiel-Aleksander 2016: 270).

Wie Marton und Booth (1997: 111) betonen, versteht sich die Phänomenographie als keine Forschungsmethode, obwohl sie methodologische Aspekte aufgreift; sie ist eher ein Weg - ein Ansatz, mit dem versucht wird, Probleme zu identifizieren, Fragen zu formulieren und diese zu beantworten, die mit dem Wesen der Lernprozesse im engen Zusammenhang stehen (vgl. auch Kallweit 2018: 138). Im Zentrum der phänomenographischen Auseinandersetzung mit der Wirklichkeit steht der Begriff Experience, dessen deutsche Pendanten Erleben / Erfahren sind. Demzufolge betrachten Marton und Booth (1997: 33) die Lernprozesse als Erleben, Erfahren von etwas: „We prefer to describe learning in terms of experience, or learning as coming to experience the world on one way or another". Diese verschiedenen Erlebensvarianten zu erfassen, ist die Aufgabe der Phänomenographie (vgl. auch Marton \& Booth 2014: 212). Wie Polat (2013: 112) zutreffend konstatiert: „,Once this variation is known, researchers will have a deeper 
understanding of what goes into learning and thinking about the phenomenon, not from the outside looking in, but hopefully from inside looking out".

Marton und Booth beziehen sich in ihren Ausführungen auf die graduelle Gliederung der Lerneraufmerksamkeit von Aron Gurwitsch (1975). Laut Gurwitsch kann das Bewusstseinsfeld in drei Ebenen der Aufmerksamkeit aufgeteilt werden:

- das THEMA: das ist der Innenhorizont - das WAS, das gerade im Fokus der Aufmerksamkeit steht - das Erlebte: die einzelnen Teile des Phänomens, die Beziehungen dieser Teile zueinander

- das thematische FELD: Kontext(e), in den / die das thematische Phänomen eingebettet ist

- den RAND: zeitliche und räumliche Aspekte, die das thematische Phänomen begleiten, aber dessen Inhalt oder Bedeutung in einer bestimmten Situation nicht tangieren (vgl. Marton \& Booth 1997; nachfolgend Kallweit 2018: 145f.).

Sowohl das thematische Feld als auch der Rand bilden den Außenhorizont. Marton und Booth schreiben insbesondere dem Gurwitschen thematischen Feld eine entscheidende Rolle zu, das ihrer Ansicht nach aus mehreren thematischen Einzelfeldern bestehen kann. Thematische Felder sind nämlich Ursache dafür, dass bestimmte Aspekte des Phänomens (etwa konkrete Situationen / Kontexte im Lernprozess) durch Lernende fokussiert werden und sich dadurch an der Konstituierung der Bedeutung, die Lernende dem Phänomen zuschreiben, beteiligen (vgl. Marton \& Booth 1997: 99; Kallweit 2018: 147). Ein Thema ist immer in einem (oder mehreren) Themenfeld(ern) verankert. Die thematischen Felder ergeben sich sowohl aus der Lerngeschichte einer Person als auch greifen sie in deren künftiges Lernen hinein (Marton \& Booth 2014: 156). Mit der Veränderung der Lernsituation können Aspekte aus dem Rand in den Fokus der Aufmerksamkeit rücken, dagegen andere periphär betrachtet werden - auf diese Art und Weise können unterschiedliche Bedeutungen eines Phänomens konstruiert werden (vgl. Kallweit 2018: 147).

Für Marton und Booth sind in der Lerneraufmerksamkeit noch zwei Aspekte wichtig: der referenzielle und der strukturelle Aspekt. Unter dem referenziellen Aspket wird das WAS des Erlebens (die dem Thema zugeschriebene Bedeutung), unter dem strukturellen Aspekt dagegen das WIE des Erlebens verstanden. Das WIE ist direkt mit dem Innen- (Thema) und Außenhorizont (Kontext und Rand) verbunden. Kallweit (2018: 150) unterstreicht: „Wenn ein Mensch etwas erlebt, erlebt er dieses etwas stets als [Herv. im Original] etwas“. Demzufolge kann die Aufmerksamkeit der Lernenden (im Sinne ihrer Struktur), jeweils von dem Kontext abhängig, auf ein Phänomen als Ganzes oder als Teile des Ganzen oder auf die Relationen zwischen den Teilen, oder zwischen den Teilen und dem Ganzen gerichtet werden. Dies kann beispielsweise mit der Frage illustriert 
werden, was konkrete Lernende unter Sprache oder aber unter Sprachenlernen verstehen. Darauf wird noch im nächsten Punkt ausführlicher eingegangen. Bilanzierend stellt Polat (2013: 113) fest: , [...] a given phenomenon may appear very differently to different learners depending of which structural element of that phenomenon the learner focuses on".

Die Grundannahme für die phänomenographische Forschung lautet, die Lernprozesse einer konkreten Person sollten nicht als ihre stabile, unveränderbare Eigenschaft angesehen werden. Sie sind eher das Ergebnis der Interpretation von Lernkontexten, wie Lernende diese erleben (vgl. Rind 2016: 2). Wie Marton und Ramsden (1988: 271; nachfolgend Rind 2016: 2) an einer anderen Stelle bemerken: „learning should be seen as a qualitative change in a person's way of seeing, experiencing, understanding, conceptualizing something in the real world".

Den phänomenographischen Untersuchungen zufolge lassen sich sechs subjektive Lernkonzeptionen unterscheiden: a) Lernen als Zunahme von Wissen, b) Lernen als Behalten und Wiedergeben, c) Lernen als Anwenden, d) Lernen als Verstehen, e) Lernen als Veränderung der Sichtweise, f) Lernen als Veränderung der Person. Während die ersten drei Konzeptionen das Lernen als Tätigkeit auffassen, tendieren die drei letzten dazu, das Lernen als Sinnstiftung zu deuten (vgl. Marton, Beaty \& Dall'Alba 1993; nachfolgend Marton \& Booth 2014: 64-67). Darüber hinaus lassen sich die ersten Lernkonzeptionen als quantitative Konzeptionen, die drei letzten hingegen als qualitative Konzeptionen bezeichnen.

Booth (1997: 137; nachfolgend Polat 2013: 114) sieht in der phänomenographischen Auseinandersetzung mit den Lernprozessen folgende vier Ziele: a) man soll den Ist- und Soll-Zustand aus den Lernerperspektiven bestimmen, b) man soll den Ist-Zustand ändern, c) man soll erfahren, wie Lernende das Lernmaterial verstehen (vgl. oben: erleben) und d) das Lehren dem Lernen anpassen.

\section{PHÄNOMENOGRAPHIE IN DER GLOTTODIDAKTISCHEN FORSCHUNG}

Im folgenden Punkt wird anhand ausgewählter Arbeiten referiert, welche Stellung der phänomenographische Ansatz in der Sprachlehr- und -lernforschung einnimmt.

Wie oben festgestellt, ist für die glottodidtkische Forschung wichtig zu ergründen, welche Konzeptionen bezüglich der Sprache an sich und fremdsprachlicher Lernprozesse bei Lernenden auftreten. Dieser Frage gehen bereits Ende der 1990er-Jahre Benson und Lor (1999) nach. Bezug nehmend auf den phänomenographischen Ansatz von Marton und Booth schlagen die Autoren eine Triade von Begriffen hinsichtlich der Lernerreflexionen über Fremdsprachenlernen vor: sie 
unterscheiden zwischen Lernkonzeptionen (conceptions), Lernermeinungen (beliefs) und Lernansätzen (approaches to learning). Lernkonzeptionen haben einen dualen Charakter: zum einen beziehen sie sich darauf, was eine Fremdsprache für konkrete Lernende ist; zum anderen - woraus die Prozesse des Fremdsprachenlernens bestehen (Benson \& Lor 1999: 465). Lernermeinungen manifestieren sich dagegen darin, was Lernende bezüglich des Phänomens Sprache und fremdsprachlicher Lernprozesse für wahr und richtig halten. Sie entstammen mehr oder weniger den Lernerfahrungen und werden in den Lernansätzen transparent. Lernansätze bilden gerade diese Ebene der Lernerreflexionen, auf der die Lernkonzeptionen und Lernermeinungen manifest werden (Benson \& Lor 1999: 471). Benson und Lor (1999: 464) stellen fest: „Approaches to learning are functional in a given context of learning and are constrained by the range of conceptions available to the learner at a given moment in time". Laut Benson und Lor (1999: 464) spiegeln die Lernkonzeptionen die höheren, abstrakteren Stufen der Lernerreflexionen als beliefs wider, sie weisen eine hierarchische Struktur auf und lassen sich auf der grundlegenden Ebene der Dichtomie quantitativ vs. qualitativ (siehe Punkt 2) beschreiben. Sowohl Lernkonzeptionen als auch beliefs sind relational zu dem gegebenen Kontext und zugleich konstituieren sich auf der Grundlage dieses Kontextes.

Die besondere Rolle des Kontextes für die Entstehung von Lernkonzeptionen und Lernermeinungen lässt sich deutlich aus den berichteten Untersuchungsergebnissen ablesen. Die von Benson und Lor befragten Studenten der Universität Hong Kong weisen mehrheitlich quantitative Lernkonzeptionen von Fremdsprachen (in diesem Fall Englisch als Fremdsprache) auf, was vor allem auf das dort geltende Prüfungssystem zurückzuführen ist (Benson \& Lor 1999: 469). In diesem Sinne bedeutet eine zu lernende Sprache für die Lernenden ein Objekt von Elementen (Grammatik, Lexik), die zu sammeln, aufzunehmen und zu beherrschen sind (Benson \& Lor 1999: 467). Die bevorzugten Lernmethoden fokussieren eine starke Rolle der Lehrenden (sie werden in der Regel als motivationale Antriebskräfte angesehen), darüber hinaus heben die Befragten die Notwendigkeit hervor, gute sprachliche Grundlagen aufzubauen und allen Elementen, die eine Sprache ausmachen, Aufmerksamkeit zu schenken (Benson \& Lor 1999: 466). Die Lernsituation wird nicht selten durch das Prisma der auftretenden Schwierigkeiten betrachtet. In Folge wirkt sich dies auch auf die beliefs about self aus: etwa dass man viel lernen muss (Benson \& Lor 1999: 467). Den quantitativen stehen einige wenige qualitative Lernansätze entgegen: im Zentrum sollen sich eigene kommunikative Bedürfnisse befinden, der Fokus soll auf den Sprachgebrauch und die Sprachumgebung gerichtet werden, in der motivationale Aspekte interner und externer Art zusammenwirken.

Benson und Lor kommen zu der Erkenntnis, dass die in dieser Studie überwiegenden quantitativen Lernansätze einen funktionalen Charakter hinsichtlich 
des beschriebenen Bildungskontextes haben. In der Tat wird aber die Disfunktionalität solcher Ansätze aufgedeckt, indem quantitativ orientierte Lernende selbst die englische Sprache als eine Bürde (im Original burden - Benson \& Lor 1999: 468) wahrnehmen, wenn sie die Sprache kommunikativ (zu) verwenden (versuchen). Benson und Lor bemerken, dass bei Lernenden mit quantitativen Lernkonzeptionen auch Aspekte qualitativer Herangehensweisen (etwa Sprachgebrauch beim Fernsehen - Benson \& Lor 1999: 470) auftreten. Das Problem liegt aber darin, dass solche Lernenden nicht immer imstande sind zu erkennen, dass sie auch dabei die Sprache lernen können. Die Lernprozesse assozieren sie nämlich nur mit institutionellen Faktoren und den daraus resultierenden quantitativen Lernkonzeptionen.

Polat (2013: 115) unterstreicht in ihren Ausführungen: „[...] phenomenography has the potential to help researchers think about traditional problems in new ways". Die bereits von Benson und Lor aufgegriffene Frage bezüglich der Spezifik des Fremdsprachenlernens („,...] participants' understanding of what second language learning is" - Herv. im Oryginal, Polat 2013: 118) befindet sich auch im Fokus dieser Studie. Am Beispiel vier amerikanischer Studierender (Religionswissenschaften, Pädagogik und Drama, 2 Personen - Angewandte Linguistik) zeigt die Autorin in Anlehnung an Marton und Booth, dass die Lernererfahrungen hinsichtlich des Sprachenlernens strukturelle und referentielle Aspekte aufweisen. Strukturelle Aspekte der Erfahrungen beziehen sich direkt auf das Begreifen des Phänomens Sprache in ihrem Wesen, Erlernen und mentalen Aufbau. Hierbei unterscheidet Polat zwischen atomistisch- und system-orientierten Lernenden (atomistic vs. systemic learners). Während die ersten die Sprache durch das Prisma einzelner Phänomene (Grammatik, Wortschatz), die man beherrschen muss, auffassen, betrachten dagegen system-orientierte Lernende die Sprache als Ganzheit, die etwa aus Grammatik und Wortschatz besteht (Polat 2013: 119). Für system-orientierte Lernende bedeuten die Lernprozesse, diese separaten Teile miteinander zu kombinieren, um die Sprache als Ganzheit im Gedächtnis aufzubauen. Referentielle Aspekte der Lernererfahrungen verweisen dagegen darauf, wozu eigentlich die Sprache für Lernende dient. Auf dieser Achse differenziert Polat zwischen extrinsischen und intrinsischen Lernenden. Extrinsische Lernende betrachten die Sprache als Kommunikationsmittel, intrinsisch orientierte Lernende - als eine Entität an sich, als etwas, was den Menschen ermöglicht, die Welt wahrzunehmen, ,[...] something that has an inherent power to influence lives and could almost be alive itself" (Polat 2013: 119).

Polat hält die strukturelle Dichotmie atomistisch- vs. system-orientierte Lernende für grundlegend, sie ordnet dieser die referentielle Dichotomie extrinsische vs. intrinsische Lernende zu. Auf diese Art und Weise entstehen vier Kategorien von Lernererfahrungen: atomistisch-extrinsisch, atomistisch-intrin- 
sisch, system-extrinsisch und system-intrinsisch. Die sich daraus ergebenden Lernererfahrungen lassen sich wie folgt charakterisieren (Polat 2013: 120-122):

a) atomistisch und extrinsisch orientierte Lernende: die Sprache besteht für sie aus separaten Elementen, die zu beherrschen sind; sie wird als Kommunikationsmittel angesehen, das nach folgendem Modell fungiert: die Elemente (etwa Wörter) können aus einer Sprache in eine andere übersetzt werden;

b) atomistisch und intrinsisch orientierte Lernende: die Sprache besteht für sie aus separaten Elementen, die zu beherrschen sind; aus referentieller Sicht wird die Sprache als Mittel verstanden, das erlaubt, die außersprachliche Wirklichkeit neu zu betrachten; wie die in der Untersuchung Befragte berichtet: „[...] it's a whole new way of redefining things” (Polat 2013: 120);

c) systemisch und extrinsisch orientierte Lernende: die Sprache wird von ihnen als ein komplexes System betrachtet, das in seinem Aufbau mit dem System einer anderen Sprache nicht immer deckungsgleich ist; die Sprache wird als Kommunikationsmittel aufgefasst; dabei zählt die Fähigkeit, dieses Kommunikationsmittel zu verwenden; die Sprachkenntnisse gelten nicht nur als sprachliches Wissen, sondern als sprachliches Können: „You have to function in that language, so you're not just learning the vocabulary or how a concept works. You need to have your passive knowledge but you also need to have your active knowledge" (Polat 2013: 121);

d) systemisch und intrinsisch orientierte Lernende: wie bereits oben dargestellt, wird die Sprache als Ganzheit aufgefasst; Fremdsprachenlernen beruht nicht darauf, dass einzelne sprachliche Bestandteile (Grammatik, Wortschatz) erlernt werden, sondern dass das System in seiner ganzen Struktur beherrscht wird; aus intrinsischer Perspektive ermöglicht die Sprache, die Welt neu zu erfahren: „It's been enlightening, sort of getting some hints of how the world is viewed by other people in other cultures..." (Polat 2013: 122).

In der Diskussion der Untersuchungsergebnisse weist Polat (2013: 122) auf die Nützlichkeit des phänomenographischen Ansatzes hin: das Kennlernen und Systematisieren der Lernererfahrungen wirft ein neues Licht auf die untersuchten Probleme, vor allem im Bereich individueller Faktoren, kognitiver Lernstile wie auch didaktischer Lösungen bei der Herstellung von Lehrmaterialien und deren Anwendung im Unterricht. Noch eine Bemerkung, die bei Polat nicht auftritt, lässt sich nach der Lektüre dieses Aufsatzes formulieren: Wie oben ausgeführt, studierten zwei von den untersuchten Lernenden Angwandte Linguistik. Gerade bei ihnen wurden systemische Lernerfahrungen, jeweils mit Subkate- 
gorien extrinsisch und intrinsisch festgestellt. Die beiden Personen studierten mehrere Fremdsprachen ( 3 bis 4 ) und hatten auch längere Aufenthalte in anderen Ländern hinter sich. Ihre Lerngeschichten können auch dazu beigetragen haben, dass sie folgende Meinungen vertreten. Die anderen zwei Lernenden (Religionswissenschaften, Pädagogik und Drama) haben offensichtlich weniger Auslandserfahrungen und keine linguistischen Fachkenntnisse, was auf ihre atomistisch ausgerichteten Lernerfahrungen und Meinungen Einfluss haben konnte. Dies erfordert aber eine weitere tiefgründere Analyse.

Die phänomenographische Untersuchungsperspektive liegt auch dem Aufsatz von Norberg, Vikström und Kirby (2018) zugrunde, in dem die Lernererfahrungen bezüglich fremdsprachlichen Wortschatzlernens (19 schwedische Lernende) im Blickpunkt des Interesses stehen. Auch diese Autoren unterstreichen das Hauptziel der phänomenographisch orientierten Forschung:

The interest of researcher is thus to study the variation [Herv. im Original] in ways of experiencing a given object or situation, not to seek evidence of an objective truth of it [...], or in the case of language, to map the process of learning, or to find out what students can produce after learning [...] (Norberg, Vikström \& Kirby 2018: 897).

Aus der Untersuchung ergibt sich, dass die Wortschatzkenntnisse in drei unterschiedlichen Perspektiven gesichtet werden können: a) als kontextloses Wissen, das verhilft, ein muttersprachliches Wort ins Englische zu übersetzen und vice versa; hier tritt offensichtlich die quantitative Konzeption des Wortschatzlernens auf; b) als kontextualisiertes Wissen - nicht nur die Bedeutungskenntnisse sind wichtig, sondern auch der Gebrauch (Lernende erkennen, dass sie manchmal die Bedeutung eines Wortes kennen, aber dieses in entsprechenden Kontexten nicht anwenden können); diese Sichtweise ist qualitativer Natur; c) als situationsbezogenes Wissen (nur wenige) - im Unterschied zu kontextualisiertem Wissen bezieht sich diese Wissensart auf konkrete Situationen, in denen Lernende Englisch als Fremdsprache gebrauchen (etwa Reisen, Berufsleben etc.). Zwei Gruppen von Lernstrategien lassen sich aus den Lernererfahrungen ableiten: a) mechanisches Wortschatzlernen (mehrheitlich) - kontextloses Memorieren auf der Grundlage von Wortschatzlisten mit muttersprachlichen Entsprechungen; b) strategisches Wortschatzlernen (nur wenige) - Lernende suchen nach Assoziationen, Kollokationen, kontextuellen Gebrauchsbeispielen etc.

Die analysierten Lernermeinungen führen Norberg et al. (2018: 902) zu der Konklusion, dass die im Bildungssystem praktizierten Lehr- / Lernweisen und Methoden der Leistungsüberprüfung starken Einfluss auf die Sichtweisen haben, aus denen sich vor allem quantitative Lernkonzeptionen ablesen lassen. 
The students repeatedly mentioned that they are given words to study each week that are most often tested in the form of translating the words, most typically from Swedish into English, as separate language items without much consideration of context. It can thus be assumed that the students' primary choice of strategy is related to the way vocabulary knowledge is assessed and how vocabulary test are constructed [...] (Norberg, Vikström \& Kirby 2018: 902).

Teilweise kritisch setzt sich mit dem phänomenographischen Ansatz Rind (2016) auseinander. Er vertritt den Standpunkt, dass die Lernweisen als Interpretation der jeweiligen Lernkontexte, in denen Lernende handeln, anzusehen sind (Rind 2016: 2). Daher sollten in die Interpretation der Lernermeinungen auch die kontextuellen Aspekte und die zwischen diesen bestehenden Relationen, die die Lernermeinungen mitkonstituieren, mehr beachtet werden. Der Forscher stellt heraus:

[...] although this approach places an emphasis on students' perceptions, it does not move behind these insights to consider the ways in which students' perceptions, conceptualization and comprehension of different variables in educational settings are also structured and shaped by contextual factors (Rind 2016: 4).

Bemägelt wird eine passive Rolle der Lernenden, wenn sie nur über ihre Erfahrungen reflektieren, diese aber von den kontextuellen Hintergründen losgelöst werden:

The learner is instead constructed as passively shaped by past experience, and passively amenable to reconstruction as a deep learner through a new set of molding processes which occur within the university: the learner [...] is "a human being without agency" (Haggis, 2003: 95) (Rind 2016: 4).

Rind schlägt daher die Miteinbeziehung der Aktivitätstheorie vor, die ursprünglich auf Vygotsky und Leontjew zurückgeführt wird und in der Gegenwart von Engeström (1987) bei der Erforschung der Lernprozesse Anwendung findet. Im klassischen Dreieck von Vygotsky haben wir drei Größen: Subjekt, Objekt / Ziel und vermittelnde Werkzeuge (tools). Im Kontext unserer Erörterungen ist der / die jeweilige Lernende ein Subjekt, das sich mit dem Objekt (hier etwa die Frage: was ist Fremdsprachenlernen?) auseinandersetzt. Als vermittelnde Werkzeuge gelten hier z.B. Sprache(n) (Muttersprache, Fremdsprache), in der / denen darüber kommuniziert wird, Medium (etwa Gespräch, E-Mail, Lerntagebuch, zwischen Lehrenden / Forschenden und Lernenden geführt). Die sich konstituierenden Beziehungen lassen sich wie folgt veranschaulichen: 
Sprache, Medium

(z.B. Gespräch, E-Mail, Lerntagebuch)

(Vermittelnde Werkzeuge)

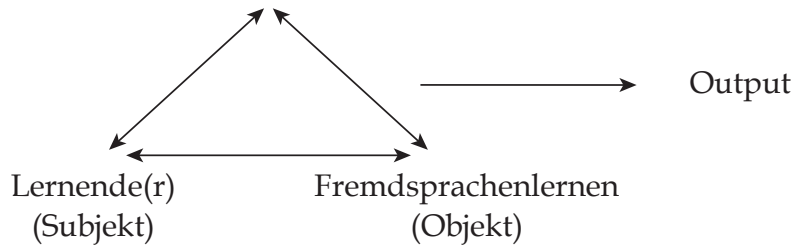

Abbildung 1. Vermittelte Beziehung zwischen Subjekt (Lerner) und Objekt (Fremdsprachenlernen)

Rind (2016: 5ff.) nimmt in dieses Modell, den theoretischen Vorschlägen von Engeström (1987) folgend, drei weitere kontextuelle Faktoren auf, die auf Aktivitäten / Handlungen der Lernenden Einfluss haben. Das sind: Gemeinschaft (community), Regeln der Gemeinschaft (rules) und Arbeitsteilung (division oflabor). Für unsere Erörterungen verstehe ich diese Relationen wie folgt: Lernende, die über ihre Erfahrungen reflektieren, handeln in bestimmten Gemeinschaften (z.B. andere Lernende, Lehrende, konkrete Gesellschaft, in der eine Fremdsprache gelernt und gebraucht wird). Selbst die Reflexionssituation (empirische Datenerhebung) ist in einer Gemeinschaft (etwa zwischen Lehrenden / Forschenden und Lernenden) situiert. In diesen Gemeinschaften herrschen bestimmte Regeln (z.B. Grad der Formalität / Informalität der Beziehungen auf der Ebene Lehrende / Forschende vs. Lernende, institutionelle Regeln). Schließlich kann die Arbeitsteilung so aufgefasst werden: ein Gespräch über Lernerfahrungen zwischen Lehrenden / Forschenden und Lernenden bildet ein Gefüge, in dem in einer reziproken Beziehung Fragen, Nach-, Klärungsfragen gestellt werden, auf die Antworten folgen. Diese rufen neue Diskussionsprobleme hervor. Modellhaft lässt sich dieses erweiterte Dreieck auf folgende Art und Weise illustrieren:

Sprache, Medium

(Gespräch, E-Mail, Lerntagebuch)

(Vermittelnde Werkzeuge)

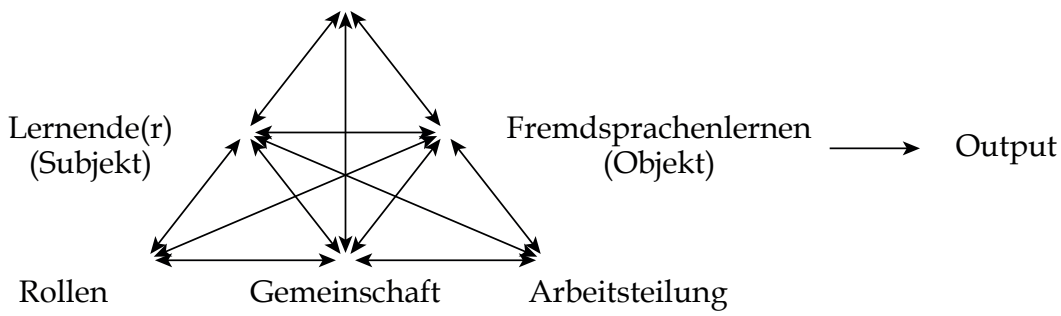

Abbildung 2. Vermittelte Beziehung zwischen Subjekt (Lerner) und Objekt (Fremdsprachenlernen) in Anlehnung an das Aktivitätsmodell von Engeström (1987) 
Wie Rind (2016:5) argumentiert: „An activity is a theoretical entity, discerned, describes, and used by the researchers to consider the socially based nature of human activity". Das dargelegte Modell veranschaulicht die Perspektive der Lernenden, die auch um die Sichtweise der Forschenden / Lehrenden, auch in einem Dreieck dargelegt, ergänzt werden kann (vgl. exemplarisch Swain, Kinnear \& Steinman 2015: 96-108). Bei der Erforschung der Lernererfahrungen soll nämlich die lehrende / forschende Person in diesem Gefüge auch als aktive(r) Handelnde(r) (Teilnehmer einer bestimmten Gemeinschaft, die ihre Regeln und Arbeitsteilung hat [etwa Lehrerstatus, Lernergruppe, in der er unterrichtet etc.] aufgefasst werden. Darüber hinaus ist wichtig hervorzuheben, dass der Fremdsprachenlehrer (als Nicht-Muttersprachler der unterrichteten Sprache) sich auch als Lerner mit seinen eigenen Erfahrungen, sowohl aus der Vergangenheit wie auch aus der Gegenwart, konfrontieren kann. Dies ist auch ein besonderer Faktor bei der Erforschung der Lernererfahrungen.

In Anlehnung an Arnold (1999: 18; nachfolgend Rind 2016:8) ist festzustellen: Fremdsprachenlernen und Gebrauch ist ", [...] a transactional process, [...] an act of reaching out beyond self to others". In unterschiedlichen Kontexten bilden Lernende unterschiedliche Identitäten: als Sprachlernende, Sprachbenutzer, Sprachmittler etc. Hierbei gehören auch ihre Rollen in den Gemeinschaften, wie sie diese definieren, welche Rollen sie den Lehrenden, Mitlernenden sowie anderen Kommunikationspartnern (nicht nur in der Fremdsprache - etwa Familie, Bekannte) zuschreiben.

\section{MÖGLICHKEITEN UND PERSPEKTIVEN DER ANWENDUNG DES PHÄNOMENOGRAPHISCHEN ANSATZES IM POLNISCHEN BILDUNGSKONTEXT}

Der dargestellte phänomenographische Ansatz liefert, unter Berücksichtigung theoretischer Prämissen der Aktivitätstheorie, für die Erforschung fremdsprachlicher Lernprozesse einen relevanten Beitrag. An dieser Stelle nenne ich einige Probleme, die im Kontext der Ausbildung von polnischen Neuphilologiestudenten weiterer Forschung bedürftig sind und aus phänomenograpischer Perspektive angegangen werden können.

Das Studieren einer Fremdsprache bildet ein besonderes Gefüge, in dem die Lernprozesse stattfinden. Zum einen wird die Sprache, vor allem auf einem fortgeschrittenen Niveau, (weiter)gelernt, zum anderen werden im Studium auch fachspezifische Kenntnisse und Fähigkeiten (etwa im Bereich der Literatur-, Kultur- und Sprachwissenschaft) erworben. Die Studienanfänger haben unterschiedliche Sprachlernerfahrungen und hegen auch verschiedene Erwartungen 
bezüglich des Studiums (vgl. auch Quetz 1998). Nicht selten fokussieren ihre Pläne und Erwartungen vor allem die Erweiterung der Sprachkenntnisse, literatur- und sprawissenschaftliche Aspekte des Studiums interessieren manche Studierenden dagegen weniger. Selbst die Sprachkurse im Studium können sich von den typischen schulischen Lehrwerkkursen unterscheiden, indem mehr Eigeninitiative und selbstständige Arbeit, insbesondere im Bereich mündlicher und schriftlicher Sprachproduktionen, erwartet werden. Vor diesem Hintergrund sind manchmal Meinungen der Studierenden zu hören, dass ihre Sprachkenntnisse während des Studiums, vor allem im Bereich der Alltagssprache, kaum gestiegen seien.

Wie oben erwähnt, haben die Studienanfänger unterschiedliche Sprachlernerfahrungen, die etwa darauf zurückzuführen sind, wie sie die Sprachen, die sie jetzt studieren möchten, bisher gelernt haben: nur institutionell (Schulunterricht, Sprachkurse etc.) und / oder auch unter natürlichen Bedingungen (Immersionsprogramme, Auslandsaufenthalte etc.). Auf diesen Sprachlernerfahrungen gründen ihre allgemeinen und fremdsprachenspezifischen Lernkonzeptionen (qualitativer vs. quantitativer Art) und konkrete Meinungen, wie Sprachen gelernt werden (können / sollen). Eine besondere Rolle kommt dabei dem schulischen Fremdsprachenunterricht $\mathrm{zu}$, in dem den Lernenden, mit mehr oder weniger bewusster Aufmerksamkeitsfokussierung, bestimmte Lern- und Kommunikationsstrategien - etwa zur Wortschatz- oder Grammatikarbeit, zum Umgang mit Fehlern (darunter auch die Einstellungen den unvermeidlichen Fehlern gegenüber) vermittelt wurden. Darüber hinaus spielen die Emotionen eine gewichtige Rolle. Die Hemmungen vor Sprachgebrauch, die sich nicht selten wegen verschiedener individueller und sozialer Ursachen zu Sprechängsten entwickelt haben, sind nur ein Beispiel dafür, dass es forschungsrelevant ist, den Lernerfahrungen und Meinungen der Studienanfänger nachzukommen (vgl. exemplarisch Nerlicki 2011: 286-293; Nerlicki \& Riemer 2012).

Solch ein festgestellter Ist-Zustand der Lernerfahrungen und Meinungen am Anfang des Studiums kann eine Grundlage für die Monitorierung möglicher Veränderungen während des Studiums bilden. Unzweifelhaft sind solche Veränderungen im Bereich der Meinungen, was Sprache ist und wie ihre Lernprozesse verlaufen (können), und schließlich, ob und wie die eigenen Lernkonzeptionen hinsichtlich des Sprachenlernens gewissen Modifikationen unterliegen sollten, notwendig. Umso wichtiger ist dies bei diesen Studierenden, die in ihrer späteren Berufspraxis Deutsch unterrichten möchten, zumal sie sich auch die Frage stellen sollten, was für ein Fremdsprachenlehrerbild aus ihren eigenen Lernerfahrungen hervorgeht und was sie davon für ihre Lehrtätigkeit (nicht) behalten werden. Im Kontext des Neuphilologiestudiums sind auch sprachenübergreifende Erfahrungen und Meinungen von Studierenden unterschiedlicher Sprachen forschungsrelevant. 


\section{ABSCHLIESSENDE BEMERKUNGEN}

Der phänomenographische Ansatz ermöglicht individuelle Lernwege und Lernermeinungen kennen zu lernen und diese auch den Lernenden bewusst zu machen. Neben forschungsorientierten Zielen kann man nämlich den Datensammlungsmethoden (etwa in Form von semistrukturierten Interviews oder Lerntagebüchern) ihre bewusstheitsfördernde und emanzipatorische Funktion nicht verkennen. Durch die Fokussierung und Auseinandersetzung mit eigenen, aber auch fremden Lerngeschichten und -meinungen werden Studierende ihrer Lernprozesse bewusster und beim Lernen und Gebrauch von Fremdsprachen selbstständiger.

\section{LITERATURVERZEICHNIS}

Adamczak-Krysztofowicz, S. (2009). Fremdsprachliches Hörverstehen im Erwachsenenalter. Theoretische und empirische Grundlagen zur adressatengerechten und integrativen Förderung der Hörverstehenskompetenz am Beispiel Deutsch als Fremdsprache in Polen. Poznań: Wydawnictwo Naukowe UAM.

Aleksandrzak, M. (2018). Rozwijanie sprawności mówienia w kształceniu neofilologicznym z perspektywy studentów uczących się języka angielskiego jako obcego. Poznań: Wydawnictwo Naukowe UAM.

Arnold, J. (1999). Affect in language learning. Cambridge: Cambridge University Press.

Benson, Ph. / Lor, W. (1999). Conceptions of language and language learning. System, 27, 459-472. Booth, S. (1997). On phenomenography, learning, and teaching. Higher Education Research and Development, 16, 135-158.

Deters-Philipp, A.-C. (2018). Lehrersprache im Englischunterricht an deutschen Grundschulen. Eine Interviewstudie mit Lehrkräften. München: Waxmann.

Ellis, M. (2014). English lessons in lower secondary schools in Poland: Reflections based on learner voices. Neofilolog, 42/1, 25-38.

Engeström, Y. (1987). Learning by expanding. An activity-theoretical approach to developmental research. Helsinki: Orienta-Konsultit.

Grotjahn, R. (1998). Subjektive Theorien in der Fremdsprachenforschung. Methodologische Grundlagen und Perspektiven. Fremdsprachen Lehren und Lernen, 27, 33-59.

Gurwitsch, A. (1975). Das Bewusstseinsfeld. Berlin / New York: De Gruyter.

Haggis, T. (2003). Constructing images of ourselves? A critical investigation into "approaches to learning" research in higher education. British Educational Research Journal, 29, 89-104. DOI: $10.1080 / 0141192032000057401$.

Jaroszewska, A. (2014). Badania biograficzne jako źródło refleksji nad procesami nauczania / uczenia się języków obcych. Neofilolog, 42/1, 51-61.

Jurgiel-Aleksander, A. (2016). Fenomenografia i jej poznawcze konsekwencje w świetle projektu na temat doświadczeń edukacyjnych dorosłych. Refleksja badacza. Rocznik Andragogiczny, 23, 267-282. DOI: 10.12775/RA.2016.014

Kalaja, P. / Barcelos, A. M. F. (2003) (Hrsg.). Beliefs about SLA. New research approaches. New York: Springer.

Kalaja, P. / Barcelos, A. M. F. / Aro, M. / Ruohotie-Lyhty, M. (Hrsg.) (2016). Beliefs, agency and identity in foreign language learning and teaching. Basingstoke / New York: Palgrave Macmillan. 
Kallenbach, Ch. (1996). Subjektive Theorien. Was Schüler und Schülerinnen über Fremdsprachenlernen denken. Tübingen: Narr.

Kallweit, N. (2018). Kindliches Erleben von Krieg und Frieden. Eine phänomenographische Untersuchung im politischen Lernen des Sachunterrichts. Wiesbaden: Springer Spektrum.

Klippel, J. (2013). Sprachlernsituation Auslandsstudium. Eine qualitative Studie zu Lernerfahrungen ausländischer Studierender in Deutschland. Baltmannsweiler: Schneider Verlag Hohengehren.

Kossobucka, A. (2013). Fenomenografia jako metoda diagnozy genetycznej. In: B. Niemierko / M. K. Szmigel (Hrsg.). Polska edukacja w świetle diagnoz prowadzonych z różnych perspektyw badawczych. Materiały XIX Konferencji Diagnostyki Edukacyjnej. Gniezno, 26-28 września 2013 r. (S. 409-412). Kraków: Tomami.

Marton, F. (1981). Phenomenography - describing conceptions of the world around us. Instructional Science, 10, 177-200.

Marton, F. / Beaty, E. / Dall'Alba, G. (1993). Conceptions of learning. International Journal of Educational Research, 19, 277-230.

Marton, F. / Booth, S. (1997). Learning and awareness. Mahwah / New Jersey: Lawrence Erlbaum.

Marton, F. / Booth, S. (2014). Lernen und Verstehen. Berlin: Logos.

Marton, F. / Ramsden, P. (1988). What does it take to improve learning? In: P. Ramsden (Hrsg.). Implying learning: New perspectives (S. 268-286). London: Kogan Page.

Michońska-Stadnik, A. (2013). Teoretyczne i praktyczne podstawy weryfikacji wybranych teorii subiektywnych w ksztatceniu nauczycieli języków obcych. Wrocław: Wydawnictwo Uniwersytetu Wrocławskiego.

Nerlicki, K. (2011). Lernerreflexionen im Blickpunkt. Polnische Germanistikstudenten über ihre fremdsprachlichen Lern- und Kommunikationserfahrungen. Hamburg: Dr. Kovač.

Nerlicki, K. / Riemer, C. (2012). Sprachverwendungsangst im interkulturellen Vergleich - auf der Suche nach ihren universellen und lernkontextspezifischen Ursachen. Deutsch als Fremdsprache, $49 / 2,88-98$.

Norberg, C. / Vikström, A. / Kirby, E. P. (2018). Secondary school students' understanding of and strategies for vocabulary acquisition: a phenomenongraphic approach to language learning. Journal of Language Teaching and Research, 9, 895-904. DOI: 10.17507/jltr.0905.02.

Pawlak, M. (2008). Advanced learners' use of strategies for learning grammar: A diary study. In: M. Pawlak (Hrsg.). Investigating English language learning and teaching (S. 109-125). Kalisz / Poznań: Faculty of Pedagogy and Fine Arts in Kalisz / Adam Mickiewicz University in Poznań.

Piechurska-Kuciel, E. (2014). Poglądy na naukę języka obcego u osób z wysoką i niską gotowością komunikacyjną w języku angielskim. Neofilolog, 42/2, 241-254.

Polat, B. (2013). Experiencing language. Phenomenography and language acquisition. Language Awareness, 22, 111-125. DOI: 10.1080/09658416.2012.658811.

Quetz, J. (1998). Mit welchen Meinungen und Einstellungen zum Englischunterricht beginnen Studierende ihr Lehramtstudium? Fremdsprachen Lehren und Lernen, 27, 106-121.

Rind, I. A. (2016). Conceptualizing students' learning experiences in English as second language in higher education from structure and agency. Cogent Social Sciences, 2, 1-16. DOI: 10.1080/23311886.2016.1191978.

Rück, N. (2009). Auffassungen vom Fremdsprachenlernen monolingualer und plurilingualer Schülerinnen und Schüler. Kassel: University Press.

Säljö, R. (1981). Learning approach and outcome: Some empirical observations. Instructional Science, 10, 47-66.

Schramm, K. / Schwab, G. (2016). Beobachtung. In: D. Caspari / F. Klippel / M. K. Legutke / K. Schramm (Hrsg.). Handbuch Forschungsmethoden in der Fremdsprachendidaktik (S. 141-154). Tübingen: Narr Francke Attempto. 
Swain, M. / Kinnear, P. / Steinman, L. (2015). Sociocultural theory in second language education. An introduction through narratives. Bristol / Buffalo / Toronto: Multilingual Matters.

Szostkiewicz, A. (2018). Sposoby doświadczania i konceptualizowania uczenia się osób w okresie średniej dorosłości. Edukacja Dorostych, 2, 163-173.

Vidgren, N. (2018). Deutsch nach Englisch und Schwedisch. Subjektive Theorien finnischer DaF-Lernender über das Lernen von mehr als einer Fremdsprache und die zwischensprachliche Interaktion. Baltmannsweiler: Schneider Verlag Hohengehren.

Werbińska, D. (2010). Teorie personalne uczniów: cztery sprawności językowe w opiniach studentów (badanie jakościowe). In: J. Knieja / T. Zygmunt / Ł. Brzana (Hrsg.). Literaturoznawstwo, jezzykoznawstwo, kulturoznawstwo jako płaszczyzny przekazu we wspótczesnej dydaktyce (S. 262-273). Lublin: Wydawnictwo UMCS.

Werbińska, D. (2015). Becoming an English language teacher: Continuities and discontinuities. Journal of Language and Cultural Education, 3, 14-31. DOI: 10.1515/jolace-2015-0002.

Received: 22.10.2019; revised: 24.03 .2020

KRZYSZTOF NERLICKI

Uniwersytet Szczeciński

krzysztof.nerlicki@usz.edu.pl

ORCID: 0000-0001-8480-1451 
